\title{
Altos niveles de adiposidad se asocian a un deterioro en la salud metabólica en adultos chilenos
}

\author{
Higher adiposity levels are associated with detrimental \\ metabolic health in Chilean adults
}

\begin{abstract}
Background: Adiposity is positively associated with metabolic and inflammatory markers, which increase the risk of developing metabolic disease related to obesity. Aim: To investigate the association between adiposity markers and metabolic health in Chilean adults. Methods: We conducted a cross-sectional study with 475 participants. Body mass index (BMI), waist circumference (WC) and body fat (using 4 skinfold) were measured. The outcomes of interest were blood pressure, fasting glucose, insulin, HOMA-IR, total cholesterol, triglycerides (TG), HDL and LDL cholesterol, $\gamma$-glutamyltransferase (CGT), alanine aminotransferase (ALT), leptin and high sensitive C-reactive protein (hsCRP). The association between adiposity and metabolic outcomes were investigated using multiple linear regression analysis. Results: Individuals in the highest tertile for BMI,WC and body fat had a lower concentration of HDL-cholesterol and higher concentration of insulin, HOMA-IR, TG, LDL and total cholesterol, GGT, ALT, leptin and hsCRP. Blood pressure was higher with increasing BMI in females only. There was no significant association between fasting glucose and any of the adiposity markers. Conclusion: Higher adiposity levels were associated with a detrimental metabolic health. The effect of higher BMI, WC and body fat were similar across metabolic markers.
\end{abstract}

Keywords: Metabolic health; cardiovascular disease; adiposity; obesity.

\section{INTRODUCCIÓN}

La obesidad se caracteriza por un incremento anormal de grasa corporal que causa un proceso inflamatorio afectando la salud ${ }^{1,2}$. Estudios epidemiológicos han demostrado una asociación positiva entre adiposidad, marcadores metabólicos y de inflamación, los que incrementan el riesgo de enfermedades metabólicas, como enfermedades cardiovasculares (ECV) y diabetes mellitus tipo $2^{3-7}$.

Existen mediciones simples de adiposidad que se usan en la clasificación y evaluación de grupos de riesgo; tales
Ana Leiva ${ }^{1}$, María Martínez², Eliana Durán ${ }^{3}$, Ana Labraña ${ }^{3}$, Ximena Díaz ${ }^{4}$, Carlos Salas ${ }^{5}$, Rodrigo Ramírez-Campillo ${ }^{6}$, Carlos Cristi-Montero ${ }^{7}$, Alex Garrido-Mendez ${ }^{8}$, Carlos Celis-Morales ${ }^{9}$.

1. Instituto de Anatomía, Histología y Patología, Facultad de Medicina, Universidad Austral de Chile, Valdivia, Chile.

2. Instituto de Farmacia, Facultad de Ciencias, Universidad Austral de Chile, Valdivia, Chile.

3. Departamento de Nutrición y Dietética, Facultad de Farmacia, Universidad de Concepción, Concepción, Chile.

4. Grupo de Investigación Calidad de Vida. Departamento de Educación. Universidad del Biobío, Chillán, Chile.

5. Departamento de Educación Física. Facultad de Educación. Universidad de Concepción. Concepción, Chile.

6. Departamento de Ciencias de la Actividad Física, Núcleo de Investigación en Salud, Actividad Física y Deporte, Universidad de Los Lagos, Osorno, Chile.

7. Grupo IRyS. Escuela de Educación Física. Pontificia Universidad Católica de Valparaíso, Valparaíso, Chile.

8. Escuela de Educación Física, Universidad San Sebastián, Concepción. 9. BHF Glasgow Cardiovascular Research Centre, Institute of Cardiovascular and Medical Science, University of Glasgow, Glasgow, United Kingdom.

Dirigir correspondencia a: Carlos Celis-Morales. BHF Glasgow
Cardiovascular Research Centre. 126 University Avenue. Glasgow
University, Glasgow. United Kingdom. G12 8TA.
Teléfono: + (0)440141 3304201.
Email: carlos.celis@glasgow.ac.uk

Este trabajo fue recibido el 25 de enero de 2017. Aceptado con modificaciones: 3 de mayo de 2017. Aceptado para ser publicado: 3 de julio de 2017.

como el índice de masa corporal (IMC), perímetro cintura (PC) y porcentaje de masa grasa (\%MG); resultando ser de utilidad en estudios epidemiológicos y práctica clínica, por ser métodos de bajo costo y fácil aplicación ${ }^{8,9}$.

El uso de marcadores de adiposidad y metabólicos, puede ser de gran utilidad en la detección temprana y reducción de riesgo de enfermedades metabólicas; así, el objetivo de este estudio fue analizar las asociaciones entre indicadores de adiposidad y marcadores metabólicos en población adulta chilena. 


\section{MATERIAL Y MÉTODO}

Diseño del estudio

Estudio observacional analítico de corte transversal, con análisis de prevalencias de factores de riesgo cardiovascular y que utiliza información del proyecto GENADIO (Genes, Ambiente, Diabetes y Obesidad), realizado entre los años 2009 al 2011, en población adulta de las regiones del Biobío y Los Ríos, Chile. Se utilizó una muestra de 475 personas para evaluar variables sociodemográficas y una sub-muestra de 370 para variables metabólicas. El cálculo del tamaño muestral se basó en diferencias HOMA-IR (homeostasis assessment model for insulin resistance), basado en estudios previos, donde se ha reportado una desviación estándar de 3-4 unidades de HOMA-IR, determinamos que se necesitarían al menos 65 participantes para cada grupo de interés (Mapuche urbano, Mapuche rural, No-Mapuche urbano, No-Mapuche rural), este tamaño permitiría detectar diferencias de 2 unidades de HOMA-IR entre estos grupos de interés. Se excluyeron del estudio mujeres en etapa de embarazo y personas con antecedentes de ECV, diabetes, hipertensión y cáncer, como también aquellas que reportaron estar bajo medicación para diabetes e hipertensión, esto ha sido descrito en extenso en publicaciones anteriores ${ }^{10,11}$. Este estudio fue aprobado por los comités de ética de las universidades de Chile, Concepción y Glasgow (Reino Unido).

Mediciones antropométricas y metabólicas

La evaluación antropométrica se realizó utilizando protocolos estandarizados ${ }^{10}$. Para determinar peso y talla se utilizó una balanza electrónica (TANITA TBF 300A, USA) y tallímetro (SECA A800, USA) con precisión de 100 g y $1 \mathrm{~mm}$ respectivamente. Para PC, se utilizó una cinta métrica no distendible (SECA, USA), con precisión de $0,1 \mathrm{~cm}$. El IMC fue calculado usando la ecuación de [peso/talla ${ }^{2}$ y el estado nutricional se definió mediante los valores de corte de la Organización Mundial de la Salud'. Para obesidad central se utilizó la medición de PC utilizando los puntos de corte: $\geq 102 \mathrm{~cm}$ hombres y $\geq 88 \mathrm{~cm}$ mujeres ${ }^{1}$. La composición corporal se determinó mediante la medición de 4 pliegues cutáneos (bicipital, tricipital, subescapular y suprailiaco), estimándose el $\%$ MG mediante la ecuación de Durnin y Womersley ${ }^{12}$. Para \%MG, se utilizaron tertiles diferenciados según sexo, (hombres: <25,7; 25,7-30,9; >30,9) y (mujeres: <29,4; $29,4-32,2 ;>32.2$ ), lo que equivale a niveles de adiposidad: bajo, medio y alto, respectivamente, para ambos sexos.

Trabajos previos ${ }^{10,11}$ describen la metodología empleada para la obtención de datos socio-demográficos y variables metabólicas como glicemia, colesterol total (CT), triglicéridos (TG), colesterol HDL (HDL-c), proteína C-reactiva ultra sensible (PCRus), alanina-amino transpeptidasa (ALT), gama-glutamil transpeptidasa (GGT), colesterol LDL (LDL-C), insulina, leptina, HOMA-IR, presión arterial diastólica (PAD) y presión arterial sistólica (PAS).

\section{Análisis de datos}

Para caracterizar variables según niveles de adiposidad se utilizó estadística descriptiva. La distribución normal de las variables fue analizada mediante el test de Anderson Darling y Kruskal-Wallis. Diferencias entre mujeres y hombres para variables continuas fueron determinadas con t-test para muestras independientes y diferencias en porcentajes entre género fue estimada mediante Chi-cuadrado. Las relaciones entre tertiles de IMC, PC y \%MG con marcadores metabólicos fueron determinadas mediante la interacción de tertiles de adiposidad y género utilizando regresión lineal múltiple y ajustando los análisis edad, sexo, nivel educacional y tabaquismo. La asociación entre marcadores metabólicos por cada 1-unidad de incremento en IMC, PC, \%MG fueron determinadas mediante regresión lineal múltiple. Valor $p$ aceptado para diferencias significativas fue $<0,05$. Todos los análisis fueron realizados con el software STATA SE 14.

\section{RESULTADOS}

La muestra quedó constituida por 475 adultos $(55,4 \%$ mujeres). La edad promedio fue 38,2 y 35,9 años para mujeres y hombres, respectivamente. En la tabla 1, se resumen las características socio-demográficas y antropométricas según sexo. La prevalencia de obesidad y marcadores de adiposidad (IMC, PC y \%MG) fueron significativamente mayores en mujeres. Al analizar la media de los indicadores de adiposidad para ambos sexos, la caracterización de la muestra corresponde a sobrepeso (según IMC), obesidad central (según PC) y nivel medio de adiposidad (según \%MG).

Las variables metabólicas caracterizadas según sexo se presentan en la tabla 2. Los niveles promedios de PCRus y LDL-c fueron significativamente mayores en mujeres, en cambio, GGT, triglicéridos y HOMA-IR fueron significativamente mayores en hombres. Para el resto de los marcadores metabólicos no se observaron diferencias significativas entre sexo.

Las asociaciones entre marcadores metabólicos y de adiposidad, según sexo, con $\mathrm{p}$ (trend) significativo, se presentan en las figuras 1, 2 y 3. Al analizar la asociación entre IMC y marcadores metabólicos (Figura 1), no se encontraron interacciones significativas entre sexo*IMC; sin embargo, los niveles de insulina, HOMA-IR, TG, colesterol total, LDL-colesterol, ALT, GGT, PCRus y leptina son significativamente mayores en ambos sexos, a un mayor IMC. Los valores de PAS y PAD son significativamente mayores en mujeres en relación a un mayor IMC. Se observa además, una concentración menor de los niveles de HDL-c en la medida que el IMC es mayor, en ambos sexos. Se encontraron resultados similares para PC y $\%$ MG (Figuras 2 y 3 ).

\section{DISCUSIÓN}

El principal hallazgo de este estudio evidencia que a mayor adiposidad, independiente del indicador empleado (IMC, PC o \%MG) se produce una tendencia al deterioro en la mayor parte de los marcadores metabólicos analizados. 
Tabla 1

Características socio-demográficas, antropométricas y actividad física según sexo.

\begin{tabular}{|c|c|c|c|}
\hline & $\begin{array}{c}\text { Mujeres } \\
\text { Prom } \pm \text { EEM }\end{array}$ & $\begin{array}{c}\text { Hombres } \\
\text { Prom } \pm \text { EEM }\end{array}$ & $p$ \\
\hline $\mathrm{n}$ & 263 & 212 & \\
\hline Edad (años) & $38,2 \pm 0,7$ & $35,9 \pm 0,9$ & 0,046 \\
\hline \multicolumn{4}{|l|}{ Nivel educacional (\%) } \\
\hline Básico & 30,6 & 30,4 & 0,158 \\
\hline Medio & 37,1 & 44,9 & \\
\hline Superior & 32,2 & 24,7 & \\
\hline \multicolumn{4}{|c|}{ Nivel socio-económico (\%) } \\
\hline Bajo & 40,8 & 43,3 & \\
\hline Medio & 33,9 & 31,4 & 0,837 \\
\hline Alto & & 25,3 & 25,3 \\
\hline Peso $(k g)$ & $68,9 \pm 0,6$ & $74,7 \pm 0,7$ & $<0,0001$ \\
\hline Talla $(\mathrm{cm})$ & $155,1 \pm 0,4$ & $164,5 \pm 0,5$ & $<0,0001$ \\
\hline IMC $\left(\mathrm{kg} \cdot \mathrm{m}^{-2}\right)$ & $28,7 \pm 0,2$ & $27,6 \pm 0,2$ & 0,003 \\
\hline \multicolumn{4}{|l|}{ Estado Nutricional (\%) } \\
\hline Normal & 17,5 & 21,7 & 0,003 \\
\hline Sobrepeso & 46,8 & 57,1 & \\
\hline Obeso & 35,7 & 21,2 & \\
\hline Masa Grasa (\%) & $30,5 \pm 0,3$ & $28,2 \pm 0,4$ & $<0,0001$ \\
\hline Perímetro cintura $(\mathrm{cm})$ & $115,1 \pm 0,7$ & $112,5 \pm 0,7$ & 0,008 \\
\hline Obesidad central (\%) & 84,4 & 35,4 & $<0,0001$ \\
\hline
\end{tabular}

\begin{tabular}{|c|c|c|c|}
\hline \multicolumn{4}{|c|}{$\begin{array}{c}\text { Tabla } 2 \\
\text { Características metabólicas según sexo. }\end{array}$} \\
\hline & $\begin{array}{c}\text { Mujeres } \\
\text { Prom } \pm \text { EEM }\end{array}$ & $\begin{array}{c}\text { Hombres } \\
\text { Prom } \pm \text { EEM }\end{array}$ & $p$ \\
\hline PAS (mmHg) & $121,4 \pm 1,1$ & $123,9 \pm 1,1$ & 0,101 \\
\hline PAD $(\mathrm{mmHg})$ & $76,0 \pm 0,7$ & $76,0 \pm 0,9$ & 0,960 \\
\hline Glicemia (mg/dl) & $98,1 \pm 1,5$ & $98,7 \pm 1,6$ & 0,790 \\
\hline Leptina (ng/ml) & $14,0 \pm 0,9$ & $13,4 \pm 0,1$ & 0,653 \\
\hline Insulina $(\mathrm{pmol} / \mathrm{l})$ & $8,0 \pm 0,6$ & $10,4 \pm 1,2$ & 0,055 \\
\hline HOMA-IR & $1,9 \pm 0,2$ & $2,5 \pm 0,3$ & 0,047 \\
\hline Colesterol total (mg/dl) & $185,9 \pm 3,4$ & $178,3 \pm 3,4$ & 0,121 \\
\hline HDL-c (mg/dl) & $35,9 \pm 1,0$ & $37,4 \pm 1,2$ & 0,328 \\
\hline LDL-c (mg/dl) & $129,4 \pm 3,7$ & $117,6 \pm 3,6$ & 0,025 \\
\hline Triglicéridos (mg/dl) & $103,8 \pm 3,8$ & $117,3 \pm 4,6$ & 0,023 \\
\hline $\operatorname{ALT}(U / I)$ & $37,8 \pm 1,5$ & $37,8 \pm 1,9$ & 0,981 \\
\hline GGT (U/I) & $32,8 \pm 1,9$ & $41,4 \pm 2,7$ & 0,008 \\
\hline PCRus (mg/l) & $1,5 \pm 0,1$ & $1,1 \pm 0,1$ & 0,007 \\
\hline
\end{tabular}



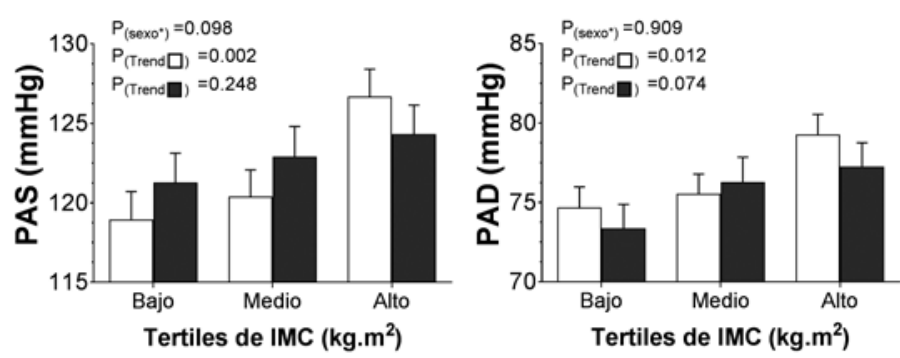

Mujeres
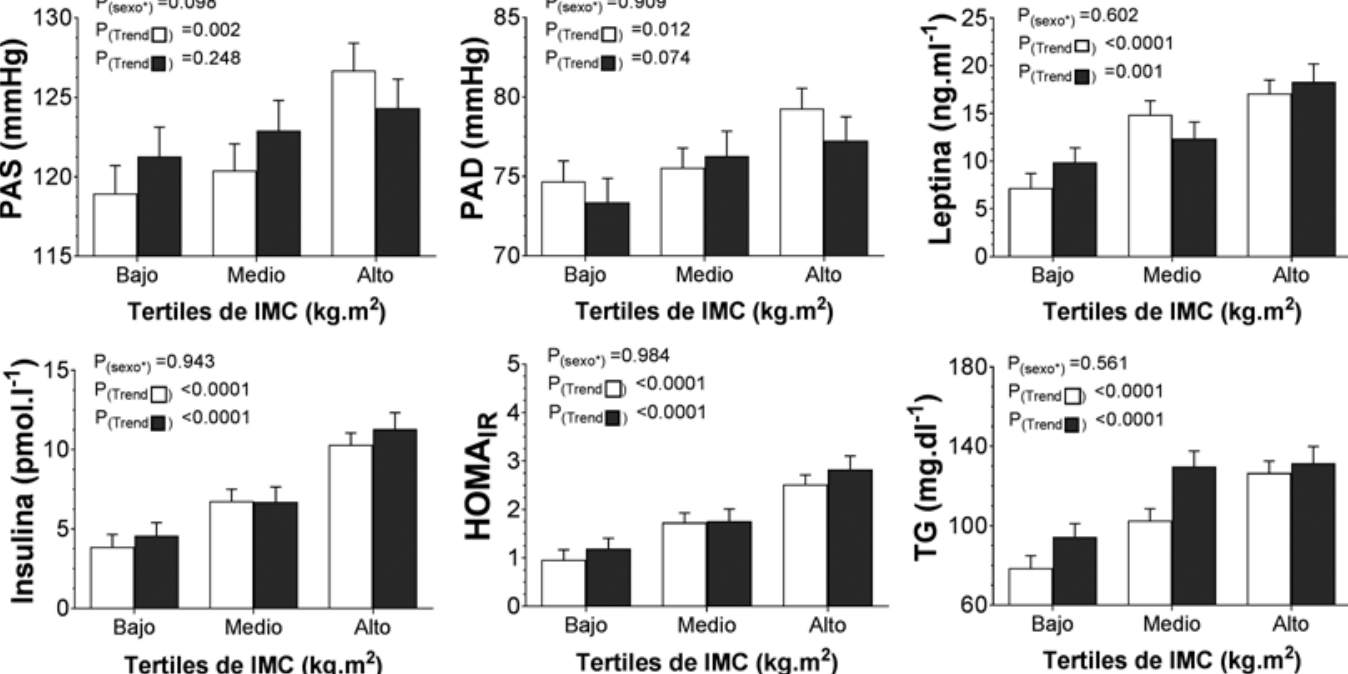

Tertiles de IMC (kg. $\left.\mathbf{m}^{2}\right)$
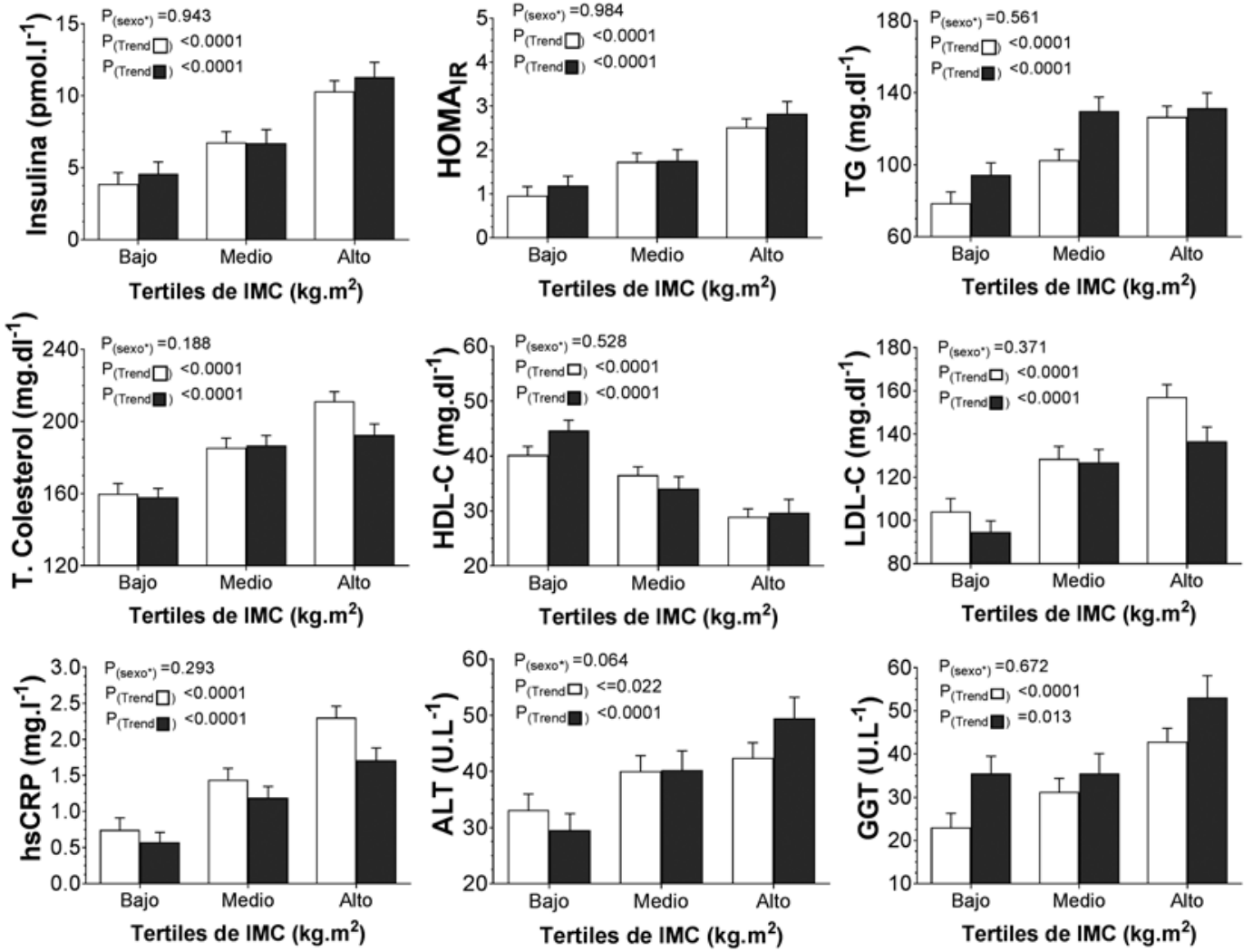

Los datos son presentados como promedio y su respectivo error estándar de la media. Los análisis fueron ajustados por edad, sexo, nivel educacional y tabaquismo. La interacción entre tertiles de IMC y sexo se estimó mediante regresión lineal y se presenta como (sexo*). El valor P-trend fue estimado para hombres y mujeres mediante análisis de regresión lineal. Los puntos de corte para tertiles de IMC fueron definidos según sexo (bajo $<26 \mathrm{~kg} / \mathrm{m}^{2}$; medio $26-29 \mathrm{~kg} / \mathrm{m}^{2}$, alto $>29 \mathrm{~kg} / \mathrm{m}^{2}$ para hombres y bajo $<27 \mathrm{~kg} / \mathrm{m}^{2} ;$ medio $27-30 \mathrm{~kg} / \mathrm{m}^{2}$, alto $>30$ $\mathrm{kg} / \mathrm{m}^{2}$ para mujeres).

Figura 1. Asociación entre marcadores metabólicos con tertiles de índice de masa corporal, según sexo. 

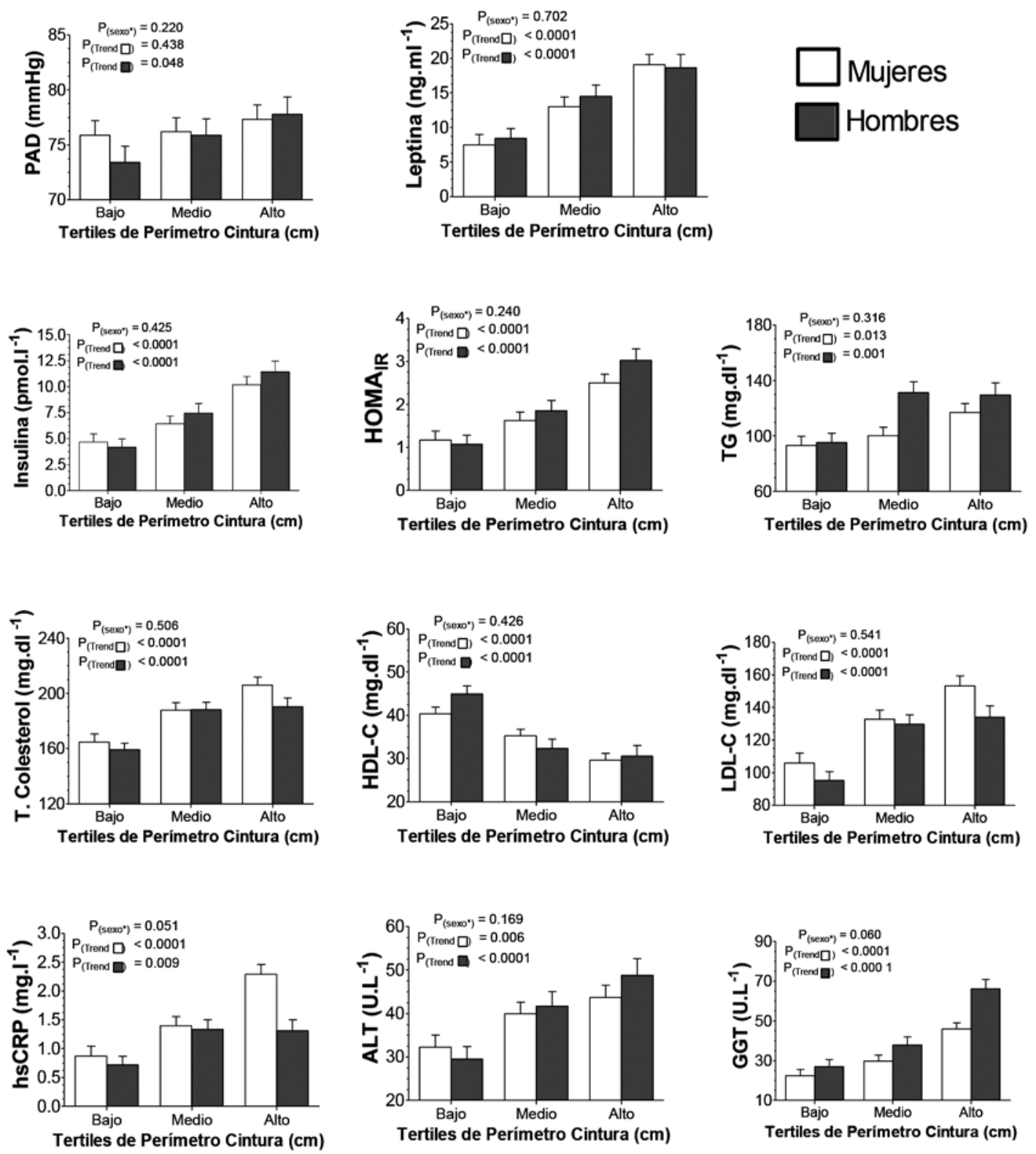

Los datos son presentados como promedio y su respectivo error estándar de la media. Los análisis fueron ajustados por edad, sexo, nivel educacional y tabaquismo. La interacción entre tertiles de Perímetro de cintura y sexo se estimó mediante regresión lineal y se presenta como (sexo*). El valor P-trend fue estimado para hombres y mujeres mediante análisis de regresión lineal. Los puntos de corte para tertiles de perímetro de cintura fueron definidos según sexo (bajo $<94 \mathrm{~cm}$; medio 94-103 cm, alto $>103 \mathrm{~cm}$ para hombres y bajo $<94 \mathrm{~cm}$; medio 94-100 cm, alto $>100 \mathrm{~cm}$ para mujeres).

Figura 2. Asociación entre marcadores metabólicos con tertiles de perímetro cintura, según sexo. 

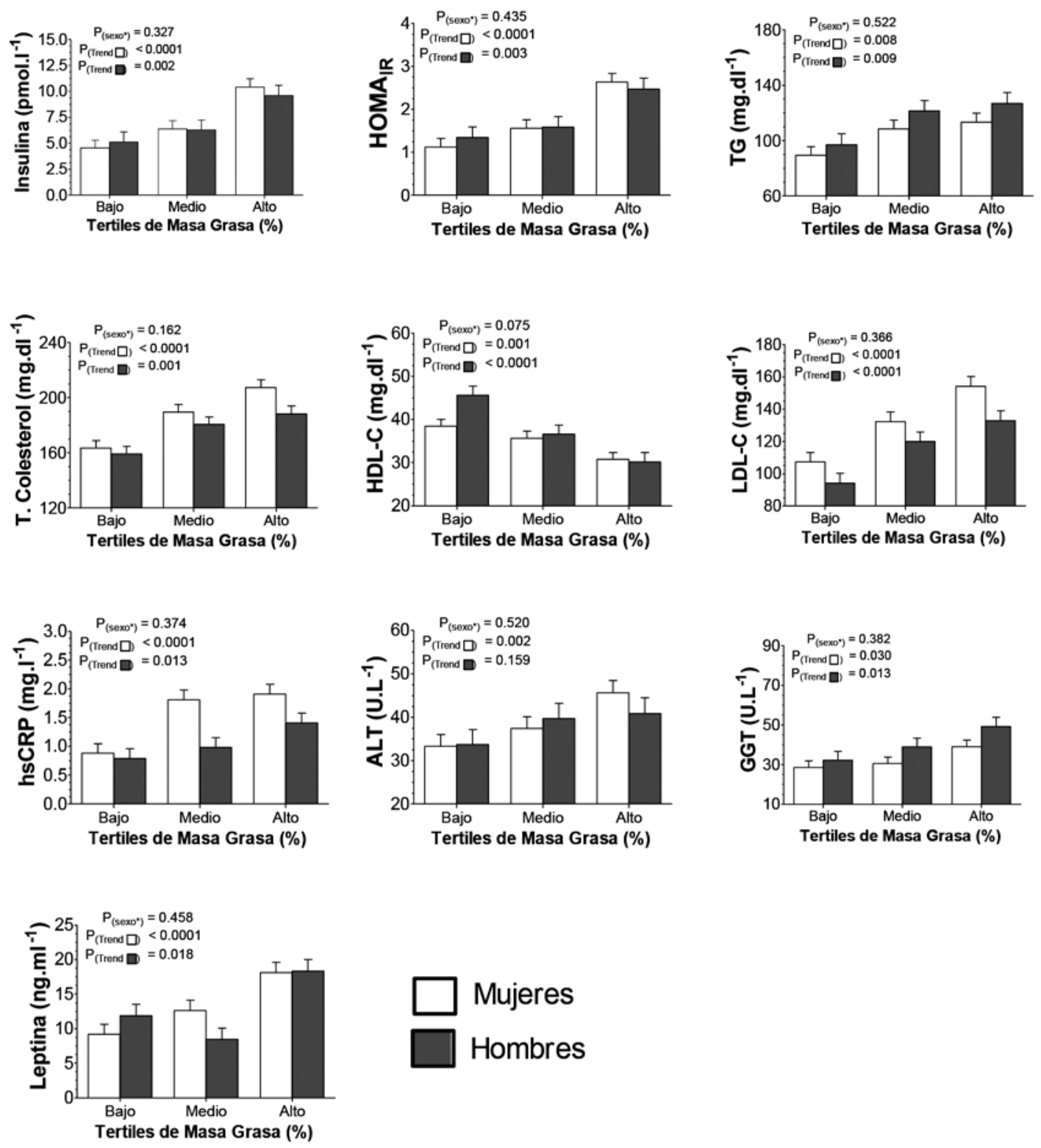

Mujeres

Hombres

Los datos son presentados como promedio y su respectivo error estándar de la media. Los análisis fueron ajustados por edad, sexo, nivel educacional y tabaquismo. La interacción entre tertiles de \% masa grasa y sexo se estimó mediante regresión lineal y se presenta como (sexo*). El valor P-trend fue estimado para hombres y mujeres mediante análisis de regresión lineal. Los puntos de corte para tertiles de masa grasa fueron definidos según sexo (bajo<25,7\%; medio 25,7-30,9\%, alto $>30,9 \%$ para hombres y bajo $<29,4 \%$; medio $29,4-32,2 \%$, alto $>32,2 \%$ para mujeres).

Figura 3. Asociación entre marcadores metabólicos con tertiles de porcentaje de masa grasa, según sexo. 
Estos resultados encontrados en una población adulta del sur de Chile, coinciden con otros trabajos realizados en otras poblaciones, que han demostrado una asociación significativa entre sobrepeso y obesidad con el riesgo de enfermedades metabólicas y ECV ${ }^{13-17}$.

La muestra presenta alta prevalencia de exceso de peso y si bien el promedio de la mayoría de los marcadores metabólicos están dentro de rangos normales, varios de los sujetos analizados presentaban valores sobre del rango normal, situación que es una alerta ya que el exceso de peso y su manifestación a través de la acumulación de tejido adiposo, constituye un importante factor de riesgo de enfermedades metabólicas ${ }^{3,18}$.

Al comparar el exceso de peso de la población en estudio con lo reportado por la Encuesta Nacional de Salud 2009-2010, se observa mayor prevalencia que la población general chilena ${ }^{19}$, lo que advierte la necesidad de desarrollar intervenciones en poblaciones del sur de Chile orientadas a cambiar sus estilos de vida.

Los tres marcadores de adiposidad estudiados mostraron que los tertiles más altos se asocian a bajos niveles de HDL-c y mayores niveles de insulina, HOMA-IR, TG, CT, LDL-C, ALT, GGT, PCRus y leptina, en ambos sexos. Estos resultados son similares a los encontrados en otras investigaciones que evidencian asociaciones significativas entre indicadores de adiposidad y marcadores metabólicos ${ }^{13,20,21}$.

Al analizar en forma individual el comportamiento de los marcadores metabólicos frente a los indicadores de obesidad; los niveles de presión arterial son ligeramente mayores cuando los indicadores de adiposidad evaluados están en el tertil superior, lo que muestra una tendencia a desarrollar hipertensión arterial (HTA) a futuro. Estudios epidemiológicos y clínicos indican que un aumento en el IMC tiene una fuerte e independiente asociación con la presencia de HTA y que un alto porcentaje de la HTA en adultos se atribuye a la adiposidad central ${ }^{22}$.

En relación al perfil lipídico, a medida que el grado de adiposidad es mayor, se observan mayores niveles de CT, LDL-C y TG y menores niveles de HDL-C; diferencias que fueron significativas al aumentar todos los indicadores de adiposidad evaluados, resultados coincidentes con otras investigaciones ${ }^{17,23}$.

El incremento de adiposidad produce un deterioro de la tolerancia a la glucosa y de la acción insulínica, conduciendo un estado de resistencia a la insulina, que aumenta el riesgo de diabetes tipo 2 y de $\mathrm{ECV}^{5,24,25}$. Al asociar los distintos tertiles de adiposidad con la resistencia a la insulina estimada a través del HOMA-IR, ésta aumentó significativamente con el incremento de los tres indicadores de adiposidad estudiados.

La obesidad condiciona un estado inflamatorio a nivel sistémico que se manifiesta con el aumento de marcadores inflamatorios, como la PCRus y leptina ${ }^{26,27}$, cuyos niveles elevados se asocian con ECV y diabetes tipo $2^{28,29,32}$. En esta investigación, los tres indicadores de adiposidad mostraron una asociación positiva con los niveles de PCRus y leptina, resultados similares a los encontrados en otros estudios ${ }^{30,31}$.

La obesidad además representa uno de los factores de mayor riesgo para el desarrollo de enfermedad hepática, caracterizada por una elevación de ALT y GGT. En esta investigación se observaron mayores niveles de ambas transaminasas al aumentar los tres indicadores de adiposidad estudiados. Estos resultados son concordantes con un estudio en adultos jóvenes saludables, donde los niveles séricos de ALT y GGT presentaron una correlación positiva con IMC y diversos indicadores de adiposidad ${ }^{33}$.

Los hallazgos de esta investigación indican que a mayor adiposidad, se produce un deterioro de los marcadores metabólicos, evidenciándose además que no existe diferencia entre utilizar indicadores de obesidad general y central para efectos de predecir cambios metabólicos en la población y anticipar su consecuente riesgo en el desarrollo enfermedades crónicas no transmisibles.

\section{RESUMEN}

Antecedentes: El exceso de grasa corporal es uno de los principales factores de riesgo de enfermedades cardiometabólicas. Objetivo: Investigar las asociaciones entre indicadores de adiposidad y metabólicos en población adulta chilena. Métodos: Estudio observacional de corte transversal en 475 adultos, a quienes se evaluó el índice de masa corporal (IMC), perímetro cintura (PC) y porcentaje de masa grasa (\%MG). Se midió presión arterial, glicemia, insulina, HOMA-IR, colesterol total, triglicéridos, colesterol HDL y LDL, alanina-amino transpeptidasa, gama-glutamil transpeptidasa, leptina y proteína C-reactiva ultra sensible (PCRus). La asociación entre indicadores de adiposidad y marcadores metabólicos fue determinada mediante regresión lineal múltiple. Resultados: Los tertiles superiores de IMC, PC y \%MG se asociaron significativamente $(p<0,05)$ con niveles bajos de colesterol HDL y altos de insulina, HOMAIR, triglicéridos, colesterol total, colesterol LDL, ALT, GGT, PCRus y leptina; esto para ambos sexos. Se observó además que los valores de presión arterial sistólica y presión arterial diastólica, fueron significativamente mayores en mujeres en relación a un mayor IMC. Conclusión: A medida que aumentó el nivel de adiposidad, se deterioran los marcadores de salud cardiovascular y metabólica, independientemente del indicador de adiposidad empleado.

Palabras clave: Salud metabólica; enfermedad cardiovascular; adiposidad; obesidad.

\section{BIBLIOGRAFÍA}

1. WHO. Obesity: preventing and managing the global epidemic. Report of a WHO consultation. World Health Organization technical report series. 894: i-xii, 1-253. 2000.

2. Reyes M. Inflammatory characteristics of obesity. Rev Chil Nutr 2010; 37(4): 498-504.

3. Rizental P, Leite N, Lopes WA, Rosa da Silva L, Consentino $C M$, Araújo CT, et al. Association between adiposity indicators, metabolic parameters and inflammatory markers in a sample of female adolescents. Arch Endocrinol Metab 2015; 59: 325-334. 
4. García Al, Niño-Silva LA, González-Ruíz K, Ramírez-Vélez R. Body adiposity index as marker of obesity and cardiovascular risk in adults from Bogotá, Colombia. Endocrinol Nutr 2015; 62: $130-137$.

5. Verma S, Hussain ME. Obesity and diabetes: An update. Diabetes \& Metab Syndr 2017; 11(1): 73-79.

6. Fuster IJ, Ouchi N, Gokce N, Walsh K. Obesity-Induced Changes in Adipose Tissue Microenvironment and Their Impact on Cardiovascular Disease. Circ Res 2016; 118: 1786-1807.

7. Jung UJ, Choi M-S. Obesity and its metabolic complications: The Role of Adipokines and the Relationship between Obesity, Inflammation, Insulin Resistance, Dyslipidemia and Nonalcoholic Fatty Liver Disease. Int I Mol Sci 2014; 15: 6184-6223.

8. Borruel S, Moltó JF, Alpañés M, Fernández-Durán E, ÁlvarezBlasco F, Luque-Ramírez M, et al. Surrogate Markers of Visceral Adiposity in Young Adults: Waist Circumference and Body Mass Index Are More Accurate than Waist Hip Ratio, Model of Adipose Distribution and Visceral Adiposity Index 2014. PLoS ONE. 9(12): e114112.

9. González A, Ureña J, Lavielle MPD, Amancio O, Elizondo $S$, Hernández H. Comparison of anthropometric indices as predictors of cardiovascular and metabolic risk in apparently healthy population. Rev Mex Cardiol 2011; 22(2): 59-67.

10. Celis-Morales CA, Perez-Bravo F, Ibáñez L, Sanzana $R$, Hormazabal E, Ulloa N, et al. Insulin resistance in Chileans of European and indigenous descent: evidence for an ethnicity $x$ environment interaction. PLoS ONE 2011; 6(9): e24690.

11. Celis-Morales CA, Pérez-Bravo F, Ibáñez L, Salas C, Bailey ME, Gill JM. Objective vs. self-reported physical activity and sedentary time: effects of measurement method on relationships with risk biomarkers. PloS ONE 2012; 7(5): e36345.

12. Durnin J, Womersley.J. Body fat assessed from total-body density and its estimation from skidfold thickness: measurement on 481 men and women aged from 16 to 72 years. Br J Nutr 1974; 32(1): 77-97.

13. Abbasi F, Blasey Ch, Reaven GM. Cardiometabolic risk factors and obesity: does it matter whether BMI or waist circumference is the index of obesity? Am J Clin Nutr 2013; 98: 637-640.

14. Brito-Núñez NJ, Alcázar RJ. Obesity and cardiometabolic risk. Review. CIMEL 2011; 16(2): 106-113.

15. Eyzaguirre $F$, Silva $R$, Román $R$, Palacio $A$, Cosentino $M$, Vega $V$, et al. Prevalence of metabolic syndrome in children and adolescents who consult with obesity. Rev Med Chile 2011; 139: 732-738.

16. Farin HMF, Abbasi F, Reaven GM. Body mass index and waist circumference both contribute to differences in insulinmediated glucose disposal in nondiabetic adults. Am J Clin Nutr 2006; 83: 47-51.

17. Valentino G, Bustamante MJ, Orellana L, Kramer V, Duran S, Adasme $M$, et al. Body fat and its relationship with clustering of cardiovascular risk factors. Nutr Hosp. 2015; 31: 2253-2260.

18. Zhang Z-q, Deng J, He L-p, Ling W-h, Su Y-x, Chen Y-u. Comparison of Various Anthropometric and Body Fat Indices in Identifying Cardiometabolic Disturbances in Chinese Men and Women. Plos ONE 2013; 8(8): e70893.
19. Ministerio de Salud. Gobierno de Chile. Encuesta Nacional de Salud 2009-2010. MINSAL, 2010. Disponible en: http://web.minsal. cl/portal/url/item/ bcb03d7bc28b64dfe040010165012d23. pdf.

20. Ortiz B, De León LG, Carrasco CE. Anthropometric indices and its relationship with biochemical markers in women. Nutr Hosp 2015; 32: 2547-2550.

21. Pourshahidi LK, Wallace JMW, Mulhern M.S, Horigan G, Strain JJ, McSorley EM, et al. Indices of adiposity as predictors of cardiometabolic risk and inflammation in Young adults. I Hum Nutr Diet 2016; 29: 26-37.

22. Kotchen TA. Obesity-Related Hypertension: Epidemiology, Pathophysiology, and Clinical Management. Am J Hipertens 2010; 23(11): 1170-1178.

23. Espinoza M, Ruiz N, Barrios E, Reigosa A, Leal U, González JC. Cardiovascular risk profile and insulin resistance according body mass index, waist circumference and hypertriglyceridemic waist in adult subjects. Rev Med Chile 2009; 137: 1179-1186.

24. Seidell, JC. Obesity, insulin resistance and diabetes - a worldwide epidemic. Br J Nutr 2000; 83(Suppl. 1): S5-S8.

25. Cefalu WT, Bray GA, Home PD, Garvey WT, Klein S, Pi-Sunyer $X$, et al. Advances in the Science, Treatment, and Prevention of the Disease of Obesity: Ref lections From a Diabetes Care Editors' Expert Forum. Diabetes Care 2015; 38: 1567-1582.

26. Choi J, Joseph L, Pilote L. Obesity and C-reactive protein in various populations: a systematic review and meta-analysis. Obesity reviews 2013; 14: 232-244.

27. Bastard JP, Maachi M, Lagathu C, Kim MJ, Caron M, Vidal H, et al. Recent advances in the relationship between obesity, inflammation, and insulin resistance. Eur. Cytokine Netw 2006; 17(1): 4-12.

28. Farooq W, Farwa U, Khan FR. The Metabolic Syndrome and Inflammation: Role of Insulin Resistance and Increased Adiposity. Oman Med J 2015; 30(2): 100-103.

29. van Wijk DF, Boekholdt SM, Arsenault BJ, Ahmadi-Abhari S, Wareham NJ, Stroes ESG, et al. C-Reactive Protein Identifies Low-Risk Metabolically Healthy Obese Persons: The European Prospective Investigation of Cancer-Norfolk Prospective Population Study. I Am Heart Assoc 2016; 5: e002823.

30. Carrión C, Barquera S, Flores M, Salmerón J, Villalpando S, Olaiz S. Association between obesity and levels of C-reactive protein as marker of chronic inflammation of low intensity in Mexican adults. Datos de la Encuesta Nacional de Salud 2000. Nutrición Clínica 2007; 10: 1-10.

31. Thompson AM, Zhang Y, Tong $W, X u T$, Chen J, Zhao L, et al. Association of obesity and biomarkers of inflammation and endothelial dysfunction in adults in Inner Mongolia, China. Int J Cardiol 2011; 150(3): 247-252.

32. Ayina CN, Noubiap JJ, Ngoa LS, Boudou P, Gautier JF, Mengnjo $M K$, et al. Association of serum leptin and adiponectin with anthropomorphic índices of obesity, blood lipids and insulin resistance in a Sub-Saharan African population. Lipids Health Dis 2016; 15: 96-106.

33. Liu X, Hamnvik OR, Chamberland JP, Petrou, Gong H, Christophi CA, et al. Circulating alanine transaminase (ALT) and $\gamma$-glutamyl transferase (GGT), but not fetuin- $A$, is associated with metabolic risk factors, at baseline and at two-year follow-up: the prospective Cyprus Metabolism Study. Metabolism 2014; 63(6): 773-782. 